\title{
Prevención y eliminación de eflorescencias en la restauración de ladrillos de construcción
}

\author{
Prevention and curing of efflorescences in the restoration of \\ bricks construction
}

\begin{abstract}
RESUMEN
Después de exponer en un artículo anterior los principios básicos de la formación del efecto eflorescente en ladrillos de construcción y definir su clasificación apoyada en algunos ejemplos, se señalan ahora los métodos para su prevención y las técnicas para su eliminación una vez aparecida esta patología. Se incluyen además algunos comentarios sobre las Normas para determinación de eflorescencias y también, de una manera muy concisa, el efecto que los gases de secaderos y hornos pueden tener en la formación de este defecto. Los métodos y técnicas de prevención y curado de eflorescencias tienen gran interés no sólo en el caso de fábricas de ladrillo modernas, sino en la restauración de fachadas de antiguos edificios.
\end{abstract}

\section{SUMMARY}

After description of basic principles of efflorescent effect in bricks in a former published paper, where the classification and examples of efflorescences were given, here are shown the methods for prevention and techniques for curing of this pathology. Some comments about Standards are included as well as very briefly the effect of gases in dryers and furnaces of efflorescences in clay products. The knowledgement of methods for prevention and curing of this pathology are very useful not only in the cleaning of modern fabrics made of bricks, but also in the Restoration of ancient buildings.

\section{INTRODUCCIÓN}

En un artículo recientemente publicado en Materiales de Construcción (1) se han expuesto, de una manera concisa, los fundamentos de la aparición del fenómeno de las eflorescencias o manchas de sales que en ocasiones aparecen en ladrillos de construcción. Se han dado los diferentes tipos de estos defectos para facilitar, así, su reconocimiento, así como ejemplos de las eflorescencias más frecuentes. El objetivo de este artículo es presentar los principales métodos para evitar la aparición de esta patología y los procedimientos básicos para su eliminación. En el caso de trabajos de restauración, estc tipo de métodos pueden ser especialmente útiles para tratar de recuperar el aspecto original de fábricas de ladrillo, tanto en edificios antiguos como modernos.

\section{EFECTO DE LOS ADITIVOS}

En el caso de los velos de secadero debe tenerse en cuenta, en primer lugar, que la cantidad de agua de amasado puede afectar fuertemente a las sales concentradas en la superficie del ladrillo. Así, un exceso de agua da lugar a mayor transporte de sales hacia la superficic (2). 
Para combatir el velo de secadero es necesario añadir sales que insolubilicen las sales que lo constituyen, $\mathrm{SO}{ }_{4} \mathrm{Ca}$ y $\mathrm{SO}_{4} \mathrm{Mg}$ principalmente. Así pues, pueden considerarse las siguientes adiciones:

$$
\begin{aligned}
& \text { - } \mathrm{SO}_{4} \mathrm{Ca}+\mathrm{CO}_{3} \mathrm{Ba} \longrightarrow \mathrm{CO}_{3} \mathrm{Ca}+\mathrm{SO}_{4} \mathrm{Ba} \\
& \text {. } \mathrm{SO}_{4} \mathrm{Ca}+\mathrm{CO}_{3} \mathrm{Na}_{2} \longrightarrow \mathrm{CO}_{3} \mathrm{Ca}+\mathrm{SO}_{4} \mathrm{Na}_{2} \\
& \text {. } \mathrm{SO}_{4} \mathrm{Mg}+2 \mathrm{PO}_{4} \mathrm{Na}_{3} \longrightarrow\left(\mathrm{PO}_{4}\right)_{2} \mathrm{Mg}_{3}+\mathrm{SO}_{4} \mathrm{Na}_{2}
\end{aligned}
$$

Hay que tener cuidado con estas adiciones, pues no deben usarse el $\mathrm{CO}_{3} \mathrm{Na}_{2}$ y el $\mathrm{PO}_{4} \mathrm{Na}_{3}$ en proporciones muy superiores al $0,25 \%$, ya que las sales sódicas alteran las condiciones de moldeo y secado de pastas cerámicas. El $\mathrm{CO}_{3} \mathrm{Ba}$ no produce alteraciones en las condiciones de trabajo, pero sí puede producir atenuación del color cuando se añade en cantidades superiores al 0,5\%. Para resolver el problema de las eflorescencias pueden ser utilizados otros aditivos, tales como:

- Cloruro bárico (1-4\%)

- Aluminato de bario y sodio

- Acido silícico coloidal

. Cloruro de sodio o calcio $(0,1-0.4 \%$ de $\mathrm{ClNa})$

- Aluminato tricálcico $(0,5-2 \%)$

. Sílice tipo "Flint" (6-12\%)

- Nitrato amónico $(0,25-0,5 \%)$

. Cloruro amónico $(0,5-2 \%)$

Para prevenir las eflorescencias, aparte de los aditivos, sobre los que volveremos más adelante, se pueden adoptar una serie de medidas tecnológicas respecto al:

- Proceso de preparación de la pasta

- Cocción del producto

En el primer caso, para mejorar la composición de la pasta se pueden usar los siguientes procedimientos:

- Usar pastas arcillosas con alto contenido en silicatos laminares.

- Evitar materias primas y aditivos que contengan pirita, derivados de azufre, marcasita o magnesio.

. Remover las capas de los denominados "fangos azules" que a menudo contienen altas cantidades de sales solubles. En el segundo caso, las medidas que pueden llegar a tomarse en el proceso de cocción son:

- Pasar con velocidad rápida el intervalo térmico desde 300 a $600^{\circ} \mathrm{C}$, aportando suficiente oxígeno.

- Eliminar los gases $\mathrm{SO}_{2}$ y $\mathrm{SO}_{3}$ que resultan de la descomposición de la pirita.

- Cocer en atmósfera reductora entre 700 y $800^{\circ} \mathrm{C}$.

- Mantener una curva de cocción de reducción de sulfatos óptima.
. Cocer a la temperatura más alta sea posible; es decir, un $0,5 \%$ por encima del reblandecimiento.

- Añadir un tiempo de mantenimiento en la temperatura máxima. Prolongar el tiempo de cocción.

- Cocer, después de la adición de manganeso, a una temperatura de al menos $1.000^{\circ} \mathrm{C}$, para evitar el lixiviado del manganeso de color marrón.

- Disminuir la superficie específica interior con un procedimiento de cocción adecuado.

Volviendo a los aditivos, revisemos en cada caso los métodos existentes para eliminar las eflorescencias de $\mathrm{SO}_{4} \mathrm{Mg}$ y las de vanadio.

\section{Eflorescencias de $\mathrm{SO}_{4} \mathrm{Mg}$}

Se pueden combatir con adiciones de:

- $\mathrm{Cl}_{2} \mathrm{Ca}$ o $\mathrm{ClNa}$ en proporciones de $0,5 \%$ y $0,1 \%$ respectivamente

- Polvo de carbón de coque (0,5-2\%)

Ceniza volante de central termoeléctrica (3\%)

- Cal en forma de polvo muy fino

- Ácido silícico coloidal al $2 \%$

Otras adiciones que se han ensayado con éxito en este tipo de eflorescencias son:

$\mathrm{CO}_{3} \mathrm{Ba}$, o $\mathrm{CO}_{3} \mathrm{Na}_{2}$ ó $\mathrm{ClNH}_{4}$ al $4 \%$

$\mathrm{Cl}_{2} \mathrm{Ca}$ al $3 \%$

$\left(\mathrm{PO}_{4}\right)_{2}\left(\mathrm{NH}_{4}\right)_{3}$ al $1 \%$

Otro método para eliminar este tipo de eflorescencias es el tratamientos de los ladrillos cocidos con siliconas. Estos tratamientos son efectivos si antes se limpia la superficie de las piezas. Se recomiendan los productos "Rhodorsyl-siliconato 51 T" del 45\% de Rhône-Poulenc (Francia). Es obvio advertir que estos tratamientos de silicona no pueden usarse en paredes que hayan sido recubiertas con mortero o yeso. Aun así, el tratamiento con siliconas ha sido puesto en tela de juicio por algunos autores, ya que argumentan que estos tratamientos podrían provocar que la cristalización se forme por debajo de la superficie, produciendo graves daños al ladrillo.

\section{Eflorescencias de Vanadio}

Se pueden prevenir por adiciones de espato fluor, $\mathrm{F}_{2} \mathrm{Ca}$, en proporciones del 1 al $4 \%$ peso. La escoria fosfórica se puede añadir en más de un $10 \%$ (3). Como medidas preventivas de tipo tecnológico se pueden recomendar (4):

- Elegir arcillas y combustibles de bajo contenido en vanadio 
. El almacenamiento de los ladrillos cocidos no debe hacerse en patios al aire libre sobre suelos de cenizas o escorias

. Aumentar la temperatura de cocción

\section{PREVENCIONES DEL FABRICANTE}

Dado que la presencia de eflorescencias va ligada directamente a los sulfatos, el principal componente que debe ser investigado tanto en el combustible como en las materias primas debe ser el azufre. El contenido en carbono, sobre todo si las materias primas (arcillas) contienen pirita, debe ser también investigado, así como el contenido de la pirita misma (2).

En el proceso de cocción, tanto la pirita como otros compuestos que contienen azufre dan lugar a reacciones que liberan gases sulfurosos que se absorberán en las superficies internas (poros) del material. Así pues, interesa prolongar el tiempo de reacción o cocción para disminuir la cantidad de gas absorbido por disminución de la superficie específica del ladrillo.

La presencia de carbón en arcillas con pirita puede ser crítica, ya que en presencia de este elemento la reacción de oxidación de la pirita puede retrasarse o aminorarse. La oxidación de la pirita suele tener lugar entre 600 y $750^{\circ} \mathrm{C}$. Sc recomienda, pues, un tratamiento térmico largo y en atmósferas limpias con abundante presencia de oxígeno.

Otra práctica recomendable es la adición a la arcilla de compuestos de bario, debido a la facilidad de los compuestos de bario de transformar los sulfatos solubles en compuestos insolubles. Hay que tener cuidado con que la adición de $\mathrm{Cl}_{2} \mathrm{Ba}$ no sea excesiva ya que él mismo puede ser eflorescente si se usa en cantidades elevadas. De la misma manera, si se añade en pequeña cantidad su efecto puede ser insuficiente. De hecho, no existe una regla o norma fija para la adición de compuestos de bario, por lo que siempre es recomendable, en este caso, hacer ensayos adicionales para determinar cuál es la cantidad óptima de sales de bario que deben añadirse a la arcilla.

Generalmente, el $\mathrm{CO}_{3} \mathrm{Ba}$ suele dar los mejores resultados, siendo incluso muy útil y más económica la adición del mineral whiterita en polvo que contiene hasta el $78-80 \%$ de este carbonato. El resto son impurezas que pueden ser convenientes para evitar el efecto de agregación de partículas. Es preciso advertir que con la adición del $\mathrm{CO}_{3} \mathrm{Ba}$ también deben tomarse precauciones y no añadirlo nunca antes del almacenamiento de la arcilla, ya que pueden producirse reacciones prematuras del carbonato con el sulfato cálcico que "agoten" la cantidad añadida de bario dejando bastante sulfato libre. Se puede añadir en polvo o bien en forma de solución y en una proporción de $8 \mathrm{~kg}$ de carbonato por tonelada de arcilla.
Adiciones de cloruros, tales como $\mathrm{ClNa}$ y $\mathrm{ClNH}_{4}$, pueden ser también utilizadas. En el caso de $\mathrm{ClNa}$ se forma $\mathrm{Cl}_{2} \mathrm{Ca}$ e incluso el ion $\mathrm{Cl}^{-}$puede actuar como mineralizador de la transformación cuarzo tridimita. Respecto al $\mathrm{ClNH}_{4}$, hay que señalar que esta sal resulta más eficaz que la anterior ya que ayuda a la descomposición de la pirita según la reacción:

$$
\mathrm{ClNH}_{4}+\mathrm{S}_{2} \mathrm{Fe} \rightarrow \mathrm{Cl}_{3} \mathrm{Fe} \uparrow+\mathrm{S}\left(\mathrm{NH}_{4}\right)_{2} \uparrow
$$

Dando lugar a la formación de compuestos que se volatilizan fácilmente.

Por ultimo, cabe mencionar que en experimentos de laboratorio (3) se ha llegado a tener éxito para hacer desaparecer las eflorescencias con adiciones de aluminatos cálcicos a las arcillas con anterioridad a su procesado.

\section{PREVENCIONES EN EL ALMACENAMIENTO}

Una vez fabricados los ladrillos, éstos deben ser almacenados, siempre que sea posible, en ausencia de humedad y nunca hacerse sobre terrenos que contengan compuestos de azufre, compuestos nitrogenados (esticrcol) o residuos orgánicos. Tampoco deberían de almacenarse sobre terrenos que lleven sustrato de escorias o residuos industriales.

\section{PREVENCIONES DEL CONSTRUCTOR}

Aunque no se considera grave la aparición de eflorescencias, de manera leve, una vez realizada la obra desapareciendo las manchas en corto espacio de tiempo, e incluso aunque aparecieran de nuevo en los ciclos anuales estacionales, el constructor tiene también una responsabilidad sobre la aparición de eflorescencias. Así, se recomienda que tome las siguientes precauciones:

a)Evitar que durante la construcción se mojen excesivamente los ladrillos.

b)Evitar que los ladrillos se contaminen con fuentes de sales solubles, ya que este tipo de contaminaciones suele dar lugar a eflorescencias muy difíciles de tratar. En este caso, son aplicables las mismas recomendaciones que para el almacenamiento .

c)Evitar la penetración anormal de agua (humedad) en los muros, sobre todo por las caras menos expuestas a la evaporación.

d)En ocasiones puede ser necesario cepillar las paredes y lavarlas con agua pura. Es preciso añadir, como recomendación vital para que no se formen eflorescencias 
en obra, que el constructor debe evitar el uso de adhesivos, morteros o aditivos que contengan azufre o sulfatos.

Métodos adicionales, y más drásticos, de tratamiento de la pared que presenta eflorescencias son:

- Limpiar con chorro de agua a presión y posterior impregnación con silicona o silanos. Ahora bien, este tipo de tratamientos puede afectar a la resistencia a la helada de los ladrillos.

. Eliminar el $\mathrm{CO}_{3} \mathrm{Ca}$ no soluble en agua, que normalmente proviene del mortero, con $\mathrm{ClH}(1: 5$ a $1: 10)$ o con preparaciones de ácido fosfórico (tipo Helotil o Murolit) (3).

- Para la eliminación de eflorescencias coloreadas de vanadio se pueden utilizar soluciones de ácido etilendiamina tetraacético (EDTA) alternadas con soluciones de $\mathrm{ClH}$, sosa caústica, o de amonio y cloruro de bario.

- Para la eliminación de eflorescencias coloreadas de hierro se pueden usar soluciones de ácido fosfórico o con mezclas de ácido acético/agua oxigenada.

\section{LIMITACIÓNDE EFLORESCENCIAS SECAS}

Ya se ha mencionado anteriormente la eficacia de adiciones de $\mathrm{CO}_{3} \mathrm{Ba}$ y $\mathrm{Cl}_{2} \mathrm{Ba}$ para combatir la presencia de sulfatos cálcicos en las materias primas, debido a las reacciones:

$$
\begin{aligned}
& \mathrm{SO}_{4} \mathrm{Ca} \\
& \text { soluble insoluble insoluble insoluble }
\end{aligned}
$$

o bien:

$$
\underset{\text { insoluble }}{\mathrm{SO}_{4} \mathrm{Ca}}+\underset{\text { soluble }}{\mathrm{Cl}_{2} \mathrm{Ba}} \rightarrow \underset{\text { insoluble }}{\mathrm{SO}_{4} \mathrm{Ba}}+\mathrm{Cl}_{2} \mathrm{Ca}
$$

debe saberse que la formación de $\mathrm{Cl}_{2} \mathrm{Ca}$ puede producir decoloraciones en el producto final. En ocasiones se han usado adiciones de fosfato sódico, aunque éstas no son muy recomendables ya que dan lugar a la formación de sulfato sódico soluble que puede ser pernicioso por su solubilidad:

$$
\underset{\text { soluble }}{\mathrm{SO}_{4} \mathrm{Ca}}+\underset{\text { soluble }}{\mathrm{PO}_{4} \mathrm{Na}_{3}} \rightarrow \underset{\text { insoluble }}{\left(\mathrm{PO}_{4}\right)_{2} \mathrm{Ca}_{3}}+\underset{\text { soluble }}{\mathrm{SO}_{4} \mathrm{Na}_{2}}
$$

Como último y original remedio se ha usado también un producto cola denominado "Perkiewicz'schen" que consigue autodepositar las sales en su estructura y luego eliminarlas al quemarse esta cola en el proceso de cocción.

\section{ELIMINACIÓN DE LAS EFLORESCENCIAS}

Se entiende por proceso de "curado" de eflorescencias a los procedimientos de lavado, raspado y/o recubrimiento que se aplican sobre una pared o productos de ladrillería "enfermos" en los que no ha sido posible prevenir o eliminar las eflorescencias durante su procesado y/o posterior puesta en obra.

Es importante tener en cuenta que en este proceso el tipo de tratamiento es específico del tipo de eflorescencia que se haya producido en la pared o muro. Y que un tratamiento no adecuado puede dar lugar a la formación de otros tipos de manchas sobre la pared. En la Tabla 1 se resume brevemente el tipo de "curado" recomendado en cada caso.

\section{VALORES LÍMITE}

\subsection{Cal de rotura}

Si la cantidad de cal de rotura presente en los ladrillos es mayor del $0,5 \%$, con partículas de diámetro mayor de $0,5 \mathrm{~mm}$ es posible la rotura del ladrillo. Así, según la norma DIN 105 (5) los ladrillos deben estar libres de oclusiones de cal de manera que no deben presentar defectos microestructurales después de ser sometidos a vapor en una proporción mayor de cinco roturas con una profundidad máxima de $3 \mathrm{~mm} / 100 \mathrm{~cm}^{2}$ de área.

\subsection{Sales solubles}

Según Lipinski (6) los límites de sulfatos de sodio y magnesio en ladrillos debe ser:

\section{. $<0,04 \%$ peso para que sean improbables las eflorescencias}

. Entre $0,04-0,08 \%$ peso para que sean posibles

. $>0,08 \%$ peso para que sean probables

Estos intervalos se cumplen también para la suma total de sulfato sódico + potásico + magnésico. Incluso, según otros autores (3), el límite de sales solubles para que no aparezcan eflorescencias está en el 0,05\%. Según la Norma DIN 105(7) diferentes productos de arcilla cocida así como los ladrillos cerámicos de alta resistencia tipo klínker deben estar libres de sales dañinas que den lugar a defectos. La Tabla 2 recoge los valores límite detectados para evitar la formación de eflorescencias después de realizar el ensayo de sales solubles según la Norma DIN $51100(8)$.

Las nuevas normas europeas al respecto resultan más específicas en cuanto al contenido de iones solubles en los ladrillos de construcción, dividiendo los límites en tres categorías de valores máximos (Tabla 3) 
TABLA 1

Curado específico para los diferentes tipos de eflorescencias

\begin{tabular}{|c|c|c|}
\hline COLOR & NATURALEZA & MÉTODO DE CURADO \\
\hline \multirow{3}{*}{ Blancas } & Sulfato & $\begin{array}{l}\text { - Cepillado y lavado con agua pura. } \\
\text { - Lavado con jabón sódico al } 1 \% \text {. }\end{array}$ \\
\hline & Carbonatos & $\begin{array}{l}\text { - Lavado con agua limpia. } \\
\text { - Lavado con ácido clorhídrico al 1:5 ó 1:10. }\end{array}$ \\
\hline & Sulfato y carbonato & $\begin{array}{l}\text { Tratamientos con Siliconas para impermeabilizar el ladrillo,pero no } \\
\text { es recomendable cuando las sales provienen del suelo. }\end{array}$ \\
\hline \multirow[t]{2}{*}{ Amarillo-Verdoso } & Vanadio & $\begin{array}{l}\text { - No lavar nunca con ácido } \mathrm{HCl} \text {. } \\
\text { - Lavar con agua destilada y solución diluida de sosa cáustica, } \mathrm{NaOH} \text {. } \\
\text { depositada sobre la superficie de los ladrillos (nunca sobre el } \\
\text { mortero) y posterior cepillado en seco (procedimiento caro, pero } \\
\text { eficaz). } \\
\text { Otro remedio es tratar la pared con soluciones de derivados del } \\
\text { EDTA (etilen-diamina-tetraacético) como el CELONE }(50 \mathrm{~g} / \mathrm{l}) \text {. }\end{array}$ \\
\hline & No vanadio & $\begin{array}{l}\text { - Si no responden al tratamiento con CELON E se recomienda usar } \\
\text { una solución de ácido acético diluido } 15 \text { veces y } \mathrm{H}_{2} \mathrm{O}_{2} \text { de } 20 \text { Vol. }\end{array}$ \\
\hline
\end{tabular}

TABLA 2

Valores límite de sales solubles para evitar la aparición de eflorescencias en ladrillos

\begin{tabular}{|c|c|c|c|}
\hline Norma DIN 105 & PRODUCTO & SALES & $\begin{array}{l}\text { VALORES LIMITES } \\
\text { MÁXIMOS (\% peso) }\end{array}$ \\
\hline PARTE 1 & $\begin{array}{l}\text { Ladrillos sólidos y perforados } \\
\text { verticalmente. Ladrillos de fachada y } \\
\text { tipo klínker. }\end{array}$ & $\begin{array}{c}\mathrm{SO}_{4} \mathrm{Mg} \\
\mathrm{SO}_{4} \mathrm{Na}_{2}+\mathrm{SO}_{4} \mathrm{~K}_{2} \\
\mathrm{SO}_{4} \mathrm{Mg}^{2} \text { adicional }\end{array}$ & $\begin{array}{l}0.12 \\
0.08 \\
0.08\end{array}$ \\
\hline PARTE 2 & $\begin{array}{l}\text { Ladrillos de alta resistencia. Ladrillos } \\
\text { de fachada y klínker. }\end{array}$ & $\begin{array}{c}\mathrm{SO}_{4} \mathrm{Mg} \\
\mathrm{SO}_{4} \mathrm{Na}_{2}+\mathrm{SO}_{4} \mathrm{~K}_{2} \\
\mathrm{SO}_{4} \mathrm{Mg} \text { adicional }\end{array}$ & $\begin{array}{l}0.12 \\
0.08 \\
0.08\end{array}$ \\
\hline PARTE 3 & $\begin{array}{l}\text { Ladrillos de alta resistencia. } \\
\text { Ladrillos de fachada y klínker. }\end{array}$ & $\begin{array}{c}\mathrm{SO}_{4} \mathrm{Mg} \\
\mathrm{SO}_{4} \mathrm{Na}_{2}+\mathrm{SO}_{4} \mathrm{~K}_{2}\end{array}$ & $\begin{array}{l}0.06 \\
0.04\end{array}$ \\
\hline PARTE 4 & Klínkers cerámicos. & $\begin{array}{c}\mathrm{SO}_{4} \mathrm{Mg} \\
\mathrm{SO}_{4} \mathrm{Na}_{2}+\mathrm{SO}_{4} \mathrm{~K}_{2}\end{array}$ & $\begin{array}{l}0.06 \\
0.04\end{array}$ \\
\hline PARTE 5 & $\begin{array}{l}\text { Ladrillos macizos ligeros. Ladrillos en } \\
\text { paneles del mismo tipo. }\end{array}$ & $\mathrm{SO}_{4} \mathrm{Mg}$ & 0.12 \\
\hline
\end{tabular}

TABLA 3

Categorias de ladrillos en cuanto a valores de sales solubles (borrador de la Norma Europea).

\begin{tabular}{|c|c|c|}
\hline TIPO ( \% peso) & IONES Na ${ }^{+}+\mathrm{K}^{+}+\mathbf{M g}^{2+}$ & IONES $\mathrm{SO}_{4}{ }^{2-}$ \\
\hline $\mathrm{L}$ (bajo) & 0.09 & 0.05 \\
\hline $\mathrm{N}$ (normal) & 0.25 & 1.6 \\
\hline $\mathrm{V}$ (no específico) & (sin condiciones) & ---- \\
\hline
\end{tabular}




\subsection{Determinación de compuestos dañinos}

Existen también las normas y procedimientos siguientes para determinar los compuestos o sales relacionados con la formación de eflorescencias en ladrillos:

Contenido en calcio. Se deduce del contenido en carbonatos determinando el $\mathrm{CO}_{2}$ por los métodos de Geiler, Shrödter u otros (2).

Inclusiones de cal. Se pueden determinar por microscopía o siguiendo la Norma DIN 105 ( $1^{\mathrm{a}}$ parte, de agosto de 1989).

\section{Sales solubles en agua}

a) Procedimiento de lixiviación por percolación según la Norma DIN 51100 de abril de 1957 que se explica con más detalle en la referencia (9).

b) Determinación cuantitativa de $\mathrm{Na}_{2} \mathrm{O}, \mathrm{K}_{2} \mathrm{O}, \mathrm{MgO}$ y CaO según la Norma DIN 51070 (de febrero de 1966, partes 1,7 y 8 ) o por espectroscopía de infrarrojo. Determinación de $\mathrm{SO}_{3}$ también según la Norma DIN 51070 o del contenido total de azufre en atmósfera oxidante entre $1.300-1.400^{\circ} \mathrm{C}$.

Tendencias a la eflorescencia. En base a los ensayos de absorción de agua y teniendo en cuenta los valores límites de las Normas DIN 105 (partes 1 a 5) y europeas (10), antes mencionadas.

\section{EFECTO DE LOS GASES SULFUROSOS EN SECADEROS Y HORNOS}

En la referencia (11) se describen los ESTUDIOS realizados por Mike y Brownell sobre el efecto de la atmósfera de $\mathrm{SO}_{2}$ en el procesado de ladrillos sobre la formación de eflorescencias. Se han hecho ensayos con atmosferas conteniendo un $0,5 \%$ y un $5 \%$ de $\mathrm{SO}_{2}$. Se han realizado adiciones del $5 \%$ de $\mathrm{CO}_{3} \mathrm{Ca}$ y $5 \%$ de $\mathrm{CO}_{3} \mathrm{Mg}$ a las materias primas y se han usado en el secadero humedades relativas de $1 \%, 2-5 \%$ y aproximadamente del $20 \%$. En estas condiciones se ha comprobado que:

a) Las atmósferas sulfurosas durante el secado y/o cocción pueden favorecer la formación de eflorescencias, sobre todo si estas atmósferas están presentes en ambos procesos.

b) Si además existe cierto grado de humedad en las materias primas utilizadas, la tendencia a la eflorescencia aumenta.

c) Asimismo, si las materias primas (arcillas) son ricas en carbonatos de calcio y/o de magnesio se favorece aun más la formación de sales de sulfatos, especialmente si existe $\mathrm{CO}_{3} \mathrm{Mg}$.

\section{CONCLUSIONES}

Los métodos y técnicas para la prevención de eflorescencias en productos de tierra cocida han sido recientemente objeto de detallada exposición por A. Verduch (12) en una amplia monografia que recoge todo los conocimientos que se tienen hasta la fecha sobre este tema de gran interés, no sólo para los fabricantes de materiales de construcción, sino también para los que aplican los ladrillos en obra. La revisión aquí presentada, a modo de manual de consulta, pretende al mismo tiempo ser un punto de partida para que a este fenómeno tan antiguo como la historia del ladrillo se le dedique una mayor atención por parte de los investigadores y un mayor esfuerzo económico en $\mathrm{I}+\mathrm{D}$ por parte de las industrias.

\section{BIBLIOGRAFÍA}

(1) Rincón, J. Ma. y Romero, M.: Materiales de Construcción (2000)

(2) García-Verduch, A.: IV Semana de Estudios Cerámicos. Ed. Soc.Esp.Ceram.Vidr. Madrid, (1967), 71-97.

(3) Schmidt, CH.: Tile and Brick International, 9(1993) 4, 199-204.

(4) García-Verduch, A.: Revista de Hyspalit (1966) 52-82.

(5)DIN 105 Teil 1 (agosto 1989): Manerziegel-Vollziegel und Hochlochziegel. Benth Verlag Gm b H, Berlin 30.

(6) Spingler, K.: Lehrbuch der Ziegeltechnik. Verlag von W. Knapp, Halle Saale, (1948), 272.

(7) García-Verduch, A.: I Congreso Iberoamericano de Cerámica, Vidrio y Refractarios, Ed. Soc.Esp.Ceram.Vidr., Madrid (1982) $577-584$.

(8) Schmidt-Reinholz, CH. and Schmidt, H.: Sprechsaal, 116 (1983) 10,878-881.

(9) Schmidt-Reinholz, CH. and Schmidt, H.: Sprechsaal, 117 (1984) 8, 692-693.

(10) Smith, R.: Tile and Brick International, 8 (1992) 1, 21-25 y 2, 100-102.

(11) AICE-ATC-IMPIVA: Tecnología de la Fabricación de Pavimentos y Revestimientos, (1990).

(12) García-Verduch, A.: Velos, Florescencias y Manchas en Obras de Ladrillo. Ed. Faenza Editrice Ibérica, Castellón, 1999. 